\title{
Pricing Catastrophe Insurance Derivatives
}

\section{By}

Alexander Muermann

DISCUSSION PAPER 400

February 2002

\section{FINANCIAL MARKETS GROUP}

AN ESRC RESEARCH CENTRE

\section{LONDON SCHOOL OF ECONOMICS}

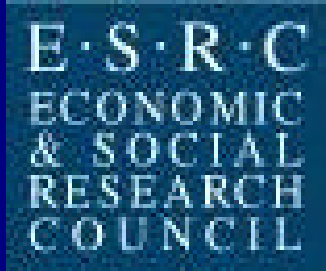

Any opinions expressed are those of the author and not necessarily those of the Financial Markets Group. 


\title{
Pricing Catastrophe Insurance Derivatives
}

\author{
Alexander Mürmann* \\ Financial Markets Group \\ and \\ The Wharton School \\ First version: October 2000 \\ This version: June 2001
}

\begin{abstract}
A bstract
We investigate the valuation of catastrophe insurance derivatives that are traded at the Chicago Board of Trade. By modeling the underlying index as a compound Poisson process we give a representation of no-arbitrage price processes using Fourier analysis. This characterization enables us to derive the inverse Fourier transform of prices in closed form for every fixed equivalent martingale measure. It is shown that the set of equivalent measures, the set of no-arbitrage prices, and the market prices of frequency and jump size risk are in one-to-one connection. Following a representative agent approach we determine the unique equivalent martingale under which prices in the insurance market are calculated.
\end{abstract}

\section{Introduction}

In recent years there has been an ongoing economic and political debate on whether financial markets should be used to insure risk that has been traditionally hedged through other channels. Famous examples include the discussion about the change to a funded pension scheme, equity-linked life insurance contracts, and insurance derivatives. This need for an alternative way of insurance resulted in a growing number of insurance products coming onto the market and containing a financial component of some sort. In order to tailor these new financial products optimally to the needs of the different markets, both finance experts as well as actuaries will have to get to know the other expert's field better. This overlap suggests that combining the methods used in both areas, insurance mathematics and mathematical finance should prove indispensable. The objective of this paper is to model the risk involved in insurance markets by the appropriate class of stochastic processes and to focus on the problem

*Insurance and Risk Management Department, The Wharton School, 306 CPC, 3641 Locust Walk, Philadelphia, PA 19104-6218; email: muermann@wharton.upenn.edu 
of price determination for catastrophe insurance derivatives that have been introduced at the Chicago Board of Trade in December 1992. These are traded financial securities based on an underlying index that encompasses insurance losses due to natural catastrophes.

Most models that have been proposed in mathematical finance include a continuity assumption on the evolution of prices, i.e. the underlying risk is predictable. In the presence of enough securities, Black and Scholes [5] and Merton [26] have shown how to determine prices of derivatives relying only on the absence of arbitrage opportunities. An arbitrage opportunity is a trading strategy that with probability one yields a positive return without any initial investment.

However, when being exposed to insurance related risk - e.g. earthquake, windstorm, or flood - one necessarily has to include unpredictable movements of the underlying index reflecting the risk involved. This leads in a natural way to the class of stochastic processes including jumps at random time points. We therefore model the dynamics of the index that underlies catastrophe insurance derivatives as a compound Poisson process, a stochastic process that is used in risk theory to model aggregate losses.

In the context of catastrophic risk, the valuation of such derivatives proves to be more problematic compared to the Black and Scholes setup [5] for two reasons. First, valuation based on arbitrage arguments make sense only when all underlying assets are explicitly defined. However, the current generation of catastrophe derivatives is based on underlying loss indices that are not traded on the market. Second, stochastic jump sizes of the underlying index 'create' an incomplete market. It is thus not possible to perfectly duplicate the movement and consequent payoffs of insurance derivatives by continuously trading in other securities. Both problems are inherently related to the fact that price processes of insurance derivatives cannot be uniquely determined solely on the basis of excluding arbitrage opportunities.

Cummins and Geman [9] were the first to investigate the valuation of catastrophe futures and derivative on futures. These securities were the first generation of traded contracts at the Chicago Board of Trade. The authors model the increments of the underlying index as a geometric Brownian motion plus a jump process that is assumed to be a Poisson process with fixed loss sizes. Because of the non-randomness in jump sizes the model can be nested into the Black and Scholes framework [5]. Since the futures' price, the basis for catastrophe derivatives, is traded on the market the market is complete and unique pricing is possible solely based on assuming absence of arbitrage opportunities. While the completeness of the market is convenient, the assumption of constant loss sizes is questionable in the context of insurance related risk. Furthermore, futures and derivatives on futures did not generate enough interest and ceased to be traded in 1995. They were replaced by options and spread options that are based on an underlying loss index that is not traded itself. The market is thus incomplete even with constant jump sizes of the underlying index.

Geman and Yor [18] examine the valuation of options that are based on the 
non-traded underlying loss index. In the paper, the underlying index is directly modeled as a geometric Brownian motion plus a Poisson process with constant jump sizes. The authors base their arbitrage arguments on the existence of a vast class of layers of reinsurance with different attachment points to guarantee completeness of the insurance derivative market. An Asian options approach is used to obtain semi-analytical solutions for call option prices in form of their Laplace transform. In addition to the assumption of constant jump sizes, the existence of a liquid catastrophe reinsurance market is questionable since coverage and premium rates in catastrophe reinsurance are individually negotiated and depend on the insurance company's past loss experience. Furthermore, the observed loss index exhibits no change in value between catastrophic events except from adjustments in loss amounts. These rare and small adjustments of the loss index do not justify dynamics with infinite variation that are inherent to a Brownian motion.

Aase [1] and [2] takes a different, more realistic modeling approach and uses a compound Poisson process with random jump sizes to describe the dynamics of the underlying index. The author investigates the valuation of catastrophe futures and derivatives on futures that ceased to be traded in 1995. Since the underlying futures' price is traded on the market the incompleteness in his setup does not arise out of the fact that the underlying index is not traded - as in Geman and Yor [18] - but from the randomness in jump sizes. The author specifies the preferences of market participants by a utility function and determines unique price processes within the framework of partial equilibrium theory under uncertainty. Closed pricing formulae are derived under the assumption of negative exponential utility function and Gamma distributed loss sizes.

In this paper, we fill the gap in the literature by investigating the valuation of current catastrophe insurance derivatives based on a non-traded underlying loss index that is modeled as a compound Poisson process with stochastic jump sizes. We therefore examine the actually traded derivatives - as in Geman and Yor [18] - while using a model that is more accurate in this actuarial context as in Aase [1] and [2].

The derivation of prices purely based on no-arbitrage arguments is very attractive as prices arise independent of investors' preferences. The disadvantage, however, is the indeterminacy of price processes since the insurance derivative market is incomplete.

In this paper, we tackle this problem in the following way. Without imposing any preferences, except that agents prefer more to less, we apply Fourier analysis to derive a representation of the class of possible price processes solely on the basis of excluding arbitrage strategies. This set of no-arbitrage price processes is parameterized by market prices of frequency and jump size risk. For every fixed pair of market prices of risk, our approach enables us to derive the inverse Fourier transform of price processes in closed form. We allow for a very general class of financial contracts - including the currently traded catastrophe derivatives and do not impose any assumptions on the distribution of jump sizes. Building upon this characterization, we show that the set of price processes excluding 
arbitrage opportunities and the set of market prices of frequency and jump size risk are one-to-one connected. In a liquid insurance derivative market, it is therefore possible to obtain the market prices of risk as implied parameters from observed derivative prices.

In the context of a market with a representative agent, market prices of frequency and jump size risk are determined by the preferences of the representative agent. The principle of utility maximization thus determines the unique price process of the insurance derivative.

An additional nice feature of our approach is that the representation of noarbitrage price processes separates the underlying stochastic structure from the financial contract's specification. The stochastic structure is captured by the characteristic function of the underlying index, the contract's specification by the inverse Fourier transform of payoffs. In a fixed stochastic environment, this separation allows for faster calculation of derivative prices. The characteristic function has to be derived once and, thereafter, the calculation of derivative prices is reduced to the derivation of the inverse Fourier transform of the contract's payoff structure.

The remainder of this paper is organized as follows: in Section 2 we discuss the catastrophe insurance market with emphasis on the current generation of catastrophe insurance options. Section 3 presents the model that describes the economic environment, the dynamics of the underlying catastrophe index and the change between equivalent probability measures. In Section 4 we investigate the pricing mechanism, first solely based on an arbitrage approach, then by adding a representative agent. Section 5 concludes.

\section{Catastrophe Insurance Derivatives}

This section presents the main ideas behind the development of the catastrophe insurance market and describes the structure and specification of existing derivatives related to catastrophic risk.

\subsection{A Iternative Risk Transfer}

The experience of major natural catastrophes in the nineties - e.g. Hurricane Andrew in 1992, Northridge California earthquake in 1994, earthquake in Kobe in 1995 - resulted in a widespread concern among insurance and reinsurance companies that there might not be enough allocated capital to meet their underwriting goals. This fear provoked a growing demand for additional capital sources and accelerated interest in using financial markets to spread catastrophic risk.

The standardization and securitization of insurance related risk provides an alternative to reinsurance contracts that traditionally have been purchased to manage catastrophe exposure. Catastrophe reinsurance is a highly customized business, where coverage and rates are individually negotiated. Premium rates 
vary depending on a specific company's present and past loss exposure, the layers covered, and current market conditions. On the contrary, financial contracts are not negotiated and contract specifications do not vary over time. In addition to the integrity and protection of standardized, exchange-traded instruments, price transparency also attracts investors and capital from outside the insurance industry.

Let us summarize the main attractions for buyers and sellers of catastrophe insurance derivatives:

First, insurance derivatives can be used by insurers and reinsurers to buy standardized protection against catastrophic risk. Alternatively, gaps in existing reinsurance contracts can be filled since financial protection can be provided between a lower desired retention level and the attachment point currently offered. In addition, these derivatives can offer an opportunity to synthetically exchange one layer for another without the need to enter costly negotiations.

Second, securitization of catastrophic risk turns catastrophes into tradeable commodities. Investors thus have the opportunity to invest indirectly in risk that traditionally has been addressed by the insurance industry only. Since catastrophic risk should prove highly uncorrelated to any other financial risk that underlies stock or bond price movements trading in catastrophes provides an additional way to diversify the investors' portfolio.

\subsection{ISO Futures and Options}

The first generation of catastrophe insurance derivatives was developed by the Chicago Board of Trade (CBoT) and trading started in December 1992. Futures and options on futures were launched based on an index that should reflect accumulated claims caused by catastrophes. The index consisted of the ratio of quarterly settled claims to total premium reported by approximately 100 insurance companies to the statistical agent Insurance Service Office (ISO). The CBoT announced the estimated total premium and the list of the reporting companies before the beginning of the trading period. A detailed description of the structure of these contracts can be found in Aase [1] and Meister [25]. Due to the low trading volume in these derivatives trading was given up in 1995 .

One major concern was a moral-hazard problem involved in the way the index was constructed: the fact that a reporting company could trade conditional on its past loss information could have served as an incentive to delay reporting in correspondence with the company's insurance portfolio. Even if the insurance company reported promptly and truthfully, the settlement of catastrophe claims might be extensive and the incurred claims might not be included in the final settlement value of the appropriate contract. This problem occurred with the Northridge earthquake which was a late quarter catastrophe of the March 1994 contract. The settlement value was too low and did not entirely represent real accumulated losses of the industry.

Since options based on these futures had more success - especially call option spreads - they were replaced by a new generation of options called PCS options. 


\subsection{PCS Catastrophe Insurance Options}

PCS Catastrophe Insurance Options were introduced at the CBoT in September 1995. They are standardized, exchange-traded contracts that are based on catastrophe loss indices provided daily by Property Claim Services (PCS) - a US industry authority which estimates catastrophic property damage since 1949. The PCS indices reflect estimated insured industry losses for catastrophes that occur over a specific period. Only cash options on these indices are available; no physical entity underlies the contracts. They can be traded as calls, puts, or spreads; futures are no longer listed for trading. Most of the trading activity occurs in call spreads, since they essentially work like aggregate excess-of-loss reinsurance agreements or layers of reinsurance that provide limited risk profiles to both the buyer and seller.

By definition, a catastrophe is an event that causes in excess of $\$ 5$ million of insured property damage and affects a significant number of policyholders and insurance companies. PCS assigns a serial number to each catastrophe for identification throughout the industry. It also compiles estimates of insured property damage using a combination of procedures, including a general survey of insurers, its National Insurance Risk Profile, and, where appropriate, its own on-the-ground survey. PCS estimates take into account both the expected dollar loss and the projected number of claims to be filed. If a catastrophe causes more than $\$ 250$ million according to preliminary estimates, PCS will continue to survey loss information to determine whether its estimate should be adjusted.

PCS Options offer flexibility in geographical diversification, in the amount of aggregate losses to be included, in the choice of the loss period and to a certain extent in the choice of the contracts' expiration date. Let us describe the contracts' specifications in more detail:

PCS provides nine geographically diverse loss indices to the CBoT: a National index; five regional indices covering Eastern, Northeastern, Southeastern, Midwestern, and Western exposures; and three state indices covering catastropheprone Florida, Texas, and California.

The CBoT lists PCS Options both as "small cap" contracts, which limit the amount of aggregate industry losses that can be included under the contract to $\$ 20$ billion, and as "large cap" contracts, which track losses from $\$ 20$ billion to $\$ 50$ billion.

Furthermore, most PCS Options track calendar quarters to allow insurers and reinsurers to focus financial coverage towards those times when they might be particularly exposed to catastrophe risk. A catastrophic event must occur during that loss period in order for resulting losses to be included in a particular index. During the loss period, PCS provides loss estimates as catastrophes occur. The PCS indices that best cover hurricane risk - Eastern, Southeastern, Florida, and Texas - all track quarterly loss periods, as do the National, Northeastern, and Midwestern indices. The California and Western indices track annual loss periods, since the catastrophe most common in that region - earth- 
quake - is not seasonal. Insurers and reinsurers that want broader protection can buy PCS Options in one-year strips, covering an entire year of risk in one transaction.

After the contract specific loss period, PCS Option users can choose either a six-month or a twelve-month development period. The development period is the time during which PCS estimates and reestimates for catastrophes that occurred during the loss period and continue to affect the PCS indices. The contract expires at the end of the chosen development period and settles in cash, even though PCS loss estimates may continue to change. The exercise style of PCS Options is European. The following table clarifies the time structure of the insurance contracts:

\begin{tabular}{|c|c|c|c|c|c|}
\hline \multirow{3}{*}{$\begin{array}{l}\text { Contract } \\
\text { M onth }\end{array}$} & \multirow{3}{*}{$\begin{array}{l}\text { Loss } \\
\text { Period }\end{array}$} & \multicolumn{2}{|c|}{ Development Period } & \multicolumn{2}{|c|}{ Settlement Date } \\
\hline & & $\mathrm{Si}$ & Twe & & Twe \\
\hline & & Month & Month & Month & Month \\
\hline Mar & Lar & & $\mathrm{Ap}$ & Sep & \\
\hline Jur & Apr-Jun & Jul 1-Dec 31 & Jul 1-Jun 30 & Dec & Jur \\
\hline September & Jul-Sep & Oct 1-Mar 31 & Oct 1-Sep 30 & Mar 31 & Sep 30 \\
\hline December & Oct-Dec & Jan 1-Jun 30 & Jan 1-Dec 31 & Jun 30 & Dec 31 \\
\hline Annual & Jan-Dec & Jan 1-Jun 30 & Jan 1-Dec 31 & Jun 30 & Dec 31 \\
\hline
\end{tabular}

Each PCS loss index represents the sum of then-current PCS estimates for insured catastrophic losses in the area and loss period divided by $\$ 100$ million. The indices are quoted in points and tenths of a point and each index point equals $\$ 200$ cash value as indicated in the chart below:

\section{PCS Loss PCS Options Industry Index Value Cash Equivalent Loss Equivalent}

$\begin{array}{lll}0.1 & \$ 20 & \$ 10 \text { million } \\ 1.0 & \$ 200 & \$ 100 \text { million } \\ 50.0 & \$ 10,000 & \$ 5 \text { billion } \\ 200.0 & \$ 40,000 & \$ 20 \text { billion (small cap limit) } \\ & & \\ 250.0 & \$ 50,000 & \$ 25 \text { billion } \\ 350.0 & \$ 70,000 & \$ 35 \text { billion } \\ 500.0 & \$ 100,000 & \$ 50 \text { billion (large cap limit) }\end{array}$

Strike values are listed in integral multiples of five points. For small cap contracts, strike values range from 5 to 195 . For large cap contracts, strike values range from 200 to 495 .

In the next section we introduce the stochastic fundamentals, the model for the dynamics of the underlying loss index, and an investigation of changing equivalent probability measures. 


\section{The Economic Environment}

Uncertainty in the insurance market is modeled by a complete probability space $(\Omega, \mathcal{F}, P)$ on which all following random variables will be defined. $\Omega$ is the set of all states of the world $\omega$ and $\mathcal{F}$ is the $\sigma$-algebra of possible events on $\Omega$. The economy has finite horizon $T<\infty$ where $T$ represents the maturity of the insurance derivative.

Let the stochastic process $X=\left(X_{t}\right)_{0 \leq t \leq T}$ represent the PCS loss index, i.e. we assume that $X_{t}$ reflects aggregated insured industry losses resulting from catastrophes up to and including time $t$. Let us suppose that all investors in this market observe the past evolution of the loss index including the current value. Therefore, the flow of information is given by the augmented filtration $\left(\mathcal{F}_{t}\right)_{0 \leq t \leq T}$ of $\sigma$-algebras generated by the process $X$ with $\mathcal{F}_{T}=\mathcal{F}$. Let us assume that the usual hypotheses hold, that is the filtration is right-continuous and $\mathcal{F}_{0}$ contains all the $P$-null sets of $\mathcal{F}$.

The market consists of one risky European insurance derivative with payoff depending on the value $X_{T}$ of the loss index at maturity $T$. We also assume the existence of a risk-free asset with price process $B=\left(B_{t}\right)_{0 \leq t \leq T}$, i.e.

$$
d B_{t}=r_{t} B_{t} d t
$$

where $r$ is the deterministic short rate of interest. Without loss of generality, we express the price process of the insurance derivative in discounted terms, i.e. we set $r \equiv 0$.

\subsection{M odeling the PCS Loss Index}

The classical approach of modeling the dynamics of financial stock prices assumes that news in the market causes an infinitesimal change in corresponding prices. Black and Scholes [5], for example, modeled the stock price as a geometric Brownian motion, i.e. as a continuous stochastic process. In actuarial risk models, however, claims cause sudden movements in the affected processes. Particularly in the context of catastrophes, losses cannot be considered as being infinitesimal. Hence we assume that catastrophic events cause unpredictable jumps in the specific PCS index at random time points. Therefore, we model the underlying index $X$ of a PCS contract by a stochastic process of the form

$$
X_{t}=\sum_{\left\{k \mid T_{k} \leq t\right\}} Y_{k}=\sum_{k=1}^{N_{t}} Y_{k},
$$

where $T_{k}$ is the random time point of occurrence of the $k t h$ catastrophe that causes a jump of size $Y_{k}$ in the underlying index and $N_{t}$ is a random variable counting catastrophic events up to time $t$. We shall assume that $X=\left(X_{t}\right)_{0 \leq t \leq T}$ is a compound Poisson process, i.e. the counting process $N=\left(N_{t}\right)_{0 \leq t \leq T}$ is a 
Poisson process with intensity $\lambda$, and $Y_{1}, Y_{2}, \ldots$ are nonnegative, independent and identically distributed random variables, all independent of the counting process $N$. Let $G$ be the distribution function of $Y_{k}$ with support $[0, \infty)$. The parameters $(\lambda, d G(y))$ are called the characteristics of the process $X$.

Under our assumption, the index $X$ of a PCS contract thus is a timehomogeneous process with independent increments. Actuarial studies (see Levi and Partrat [23]) have shown that these assumptions are reasonable in the context of losses arising from windstorm, hail and flood. Earthquakes are described as events arising from a superposition of events caused by several independent sources. The PCS index therefore approximates a compound Poisson process. The assumption on time-homogeneity is questionable for the case of hurricanes which occur seasonally. However, the indices of regions, that are exposed to hurricane risk, all track quarterly loss periods to account for seasonal effects.

Remark 1 Filtrations that are generated by compound Poisson processes and completed by $P$-null sets of $\mathcal{F}$ satisfy the usual hypotheses, i.e. they are rightcontinuous (see Protter [29] p. 22).

\subsection{Change of Equivalent $M$ easures}

In this section we examine the change between equivalent probability measures and the change in the characteristics that it induces on compound Poisson processes. We restrict the set of equivalent probability measures to the subset of probability measures under which the structure of the underlying process $X$ is preserved, i.e. under which the index remains a compound Poisson process. This subset has been characterized by Delbaen and Haezendonck [12] as follows:

Let $P$ denote the physical probability measure in the insurance market under which the compound Poisson process $X$ has characteristics $(\lambda, d G(y))$. A probability measure $Q$ is equivalent to $P$, and $X$ is a compound Poisson process under $Q$ if and only if there exists a nonnegative constant $\kappa$ and a nonnegative, measurable function $v: \mathbb{R}_{+} \rightarrow \mathbb{R}$ satisfying

$$
\int_{0}^{\infty} v(y) d G(y)=1
$$

such that the associated density process $\xi_{t}=\mathbf{E}^{P}\left[\xi_{T} \mid \mathcal{F}_{t}\right]$ of the Radon-Nikodym derivative $\xi_{T}=\frac{d Q}{d P}$ is given by

$$
\begin{aligned}
\xi_{t} & =\left(\prod_{k=1}^{N_{t}} \kappa v\left(Y_{k}\right)\right) \cdot \exp \left(\int_{0}^{t} \int_{0}^{\infty}(1-\kappa v(y)) \lambda d G(y) d s\right) \\
& =\exp \left(\sum_{k=1}^{N_{t}} \ln \left(\kappa v\left(Y_{k}\right)\right)+\lambda(1-\kappa) t\right),
\end{aligned}
$$

for any $0 \leq t \leq T . \mathbf{E}^{P}[\cdot]$ denotes the expectation operator under the probability measure $P$. 
Under the new measure $Q$ the process $X$ has characteristics $\left(\lambda^{Q}, d G^{Q}(y)\right)$ $=(\lambda \kappa, v(y) d G(y))$.

Let us denote the measure $Q$ corresponding to the constant $\kappa$ and the function $v(\cdot)$ by $P^{\kappa, v}$ and the corresponding distribution function $G^{Q}$ by $G^{v}$. Hence, for all $A \in \mathcal{B}_{+}$

$$
G^{v}(A)=\int_{A} v(y) d G(y)
$$

and

$$
\mathbf{E}^{P^{\kappa, v}}\left[N_{1}\right]=\lambda \kappa,
$$

where $\mathcal{B}_{+}$represents the Borel $\sigma$-algebra on $\mathbb{R}_{+}$and $\mathbf{E}^{P^{\kappa, v}}[\cdot]$ denotes the expectation operator under the measure $P^{\kappa, v}$.

Remark 2 In an economic sense, $\kappa$ can be interpreted as a premium of frequency risk and $v(\cdot)$ as a premium of claim size risk.

R emark 3 Meister [25] generalized the result of Delbaen and Haezendonck [12] to mixed Poisson and doubly stochastic Poisson processes.

In the following Lemma we show that the correspondence between the set of parameters $\kappa, v(\cdot)$ and the set of equivalent measures $P^{\kappa, v}$ is one-to-one.

Lemma 4 Define $K \times V \equiv\left\{(\kappa, v(\cdot)) \in \mathbb{R}_{+} \times \mathbf{L}^{1}\left(\mathbb{R}_{+}, G\right) \mid \mathbf{E}^{P}\left[v\left(Y_{1}\right)\right]=1\right\}$. Then the mapping

$$
(\kappa, v(\cdot)) \in K \times V \rightarrow P^{\kappa, v}
$$

is injective.

Proof. Let $(\kappa, v(\cdot))$ and $\left(\kappa^{\prime}, v^{\prime}(\cdot)\right)$ belong to $K \times V$ with $P^{\kappa, v}=P^{\kappa^{\prime}, v^{\prime}}$. Then $\mathbf{E}^{P^{\kappa, v}}\left[N_{1}\right]=\mathbf{E}^{P^{\kappa^{\prime}, v^{\prime}}}\left[N_{1}\right]$ and thus $\kappa=\kappa^{\prime}$. Furthermore, for all $A \in \mathcal{B}_{+}$

$$
\int_{A} v(y) d G(y)=\int_{A} v^{\prime}(y) d G(y)
$$

and so $v \equiv v^{\prime} G$-a.s.

\section{Pricing of Insurance Derivatives}

The aim of this section is to investigate the price determination of insurance derivatives that are based on PCS indices under the assumption of the previous section that the underlying index is a compound Poisson process. First, we review the equivalence between the existence of equivalent martingale measures and the absence of arbitrage opportunities in the market. Then by solely imposing absence of arbitrage possibilities we derive the inverse Fourier transform of price processes in closed form. Thereafter, we will be more restrictive and assume the existence of a representative investor in the market whose preferences determine uniquely the price of derivatives. 


\subsection{The Fundamental Theorem of Asset Pricing}

The equivalence between the existence of equivalent martingale measures and the absence of arbitrage opportunities in the market plays a central role in mathematical finance. An equivalent martingale measure is a probability measure that is equivalent to the "reference" measure $P$ and under which discounted price processes are martingales. It is important to be aware of the specifications of the model in which this equivalence is used since arbitrage has to be differently defined to guarantee the existence of equivalent martingale measures.

Harrison and Kreps [19], and Harrison and Pliska [20] were the first to establish an equivalence result in a model based on a finite state space $\Omega$. In a discrete infinite or continuous world, the absence of arbitrage is not a sufficient condition for the existence of an equivalent martingale measure. Other definitions of arbitrage opportunity or restricting conditions on the dynamics of price processes have been derived to guarantee the existence of martingale measures. Fritelli and Lakner [16] give a definition of arbitrage, called "free lunch", under which the equivalence result is derived with high level of generality. The only mathematical condition that is imposed on asset prices is that they are adapted to the filtration $\left(\mathcal{F}_{t}\right)_{0 \leq t \leq T}$ which is a natural requirement.

As asset price processes are not a priori assumed to be semimartingales stochastic integrals that reflect achievable gains from continuous trading strategies are not well-defined. To circumvent this problem, the set of trading strategies is restricted to permit trading at either deterministic times or stopping times. The "no free lunch" condition then postulates that the set of achievable gains contains no positive random variables. In a continuous time setting closure of the set of gains has to be considered which essentially depends on the topology on this set. Under a topology that makes use of certain dualities, Fritelli and Lakner [16] prove that there is "no free lunch" with trading strategies at deterministic times if and only if there exists an equivalent martingale measure. Furthermore, if every underlying process is right-continuous, then this result holds additionally for trading strategies at stopping times.

Henceforth, we assume "no free lunch" in the market as outlined above, so that the existence of an equivalent martingale measure is guaranteed.

\subsection{Representation of No-A rbitrage Prices}

In this subsection we deduce a representation of prices solely on the basis of excluding arbitrage opportunities as defined above. We will present two possible methods of deriving prices:

- the first relies on risk neutral valuation and is simply a calculation of the expected payoff under the appropriate probability measure;

- the second method makes use of the infinitesimal generator of the underlying process $X$ to derive prices as solutions of the appropriate integrodifferential equation that represents the corresponding pricing equation. 
In the catastrophe insurance market, the underlying index $X$ is not traded. Thus it is not possible to construct a hedging portfolio based on $X$ and hence the price of a derivative cannot be uniquely determined by the assumption of "no free lunch" in the market. However, assuming "no free lunch" guarantees the existence of an equivalent probability measure $Q \sim P$ under which discounted price processes of insurance derivatives are martingales. In addition, our model exhibits a second source of incompleteness arising from stochastic jump sizes of the underlying PCS index.

Let us suppose that we choose and fix an arbitrary equivalent martingale measure $Q$ such that the index process $X=\left(X_{t}\right)_{0 \leq t \leq T}$ remains a compound Poisson process after the change to the probability measure $Q$ with characteristics $\left(\lambda^{Q}, d G^{Q}(y)\right)$. The set of equivalent probability measures that preserve the structure of $X$ has been characterized by Delbaen and Haezendonck [12] and presented in Section 3.2, p. 9 of this paper.

\subsubsection{First $M$ ethod (Risk neutral valuation)}

Assuming "no free lunch" in the market, a consistent price process of an insurance derivatives that pays out $\phi\left(X_{T}\right)$ at maturity can be expressed as

$$
\pi_{t}^{Q}=\mathbf{E}^{Q}\left[\exp \left(-\int_{t}^{T} r_{s} d s\right) \phi\left(X_{T}\right) \mid \mathcal{F}_{t}\right] .
$$

$\pi_{t}^{Q}$ is of the form $f^{Q}\left(X_{t}, t\right)$ since we have assumed that $r$ is deterministic $\left(r \equiv 0\right.$ without loss of generality), $\left(\mathcal{F}_{t}\right)_{0 \leq t \leq T}$ is generated by $X$, and $X$ is a Markov process under $Q$. The stochastic process $\left(f^{Q}\left(X_{t}, t\right)\right)_{0 \leq t \leq T}$ reflects the consistent price process under the probability measure $Q$ with payoff $f^{Q}\left(X_{T}, T\right)=\phi\left(X_{T}\right)$ at maturity $T$.

Let us assume that $\phi: \mathbb{R} \rightarrow \mathbb{R}$ is a measurable function such that $\phi(\cdot)-k \in$ $\mathbf{L}^{2}(\mathbb{R})=\left\{g: \mathbb{R} \rightarrow \mathbb{C}\right.$ measurable $\left.\left.\left|\int_{-\infty}^{\infty}\right| g(x)\right|^{2} d x<\infty\right\}$ for some $k \in \mathbb{R}$. This assumption is satisfied by all catastrophe insurance derivatives that are traded at the CBoT. Notice that the payoff of all call options is capped at either $\$ 20$ billion or $\$ 50$ billion. We will now make use of Fourier analysis to calculate the expected payoff in (6).

The Fourier transformation is a one-to-one mapping of $\mathbf{L}^{2}(\mathbb{R})$ onto itself. In other words, for every $g \in \mathbf{L}^{2}(\mathbb{R})$ there corresponds one and only one $f \in \mathbf{L}^{2}(\mathbb{R})$ such that the Fourier transform of $f$ is the function $g$, that is

$$
f(u)=\frac{1}{2 \pi} \int_{-\infty}^{\infty} e^{-i u x} g(x) d x
$$

is the inverse Fourier transform of $g$. 
Applying the Fourier transform, and thereafter the inverse Fourier transform, to the function $\phi(\cdot)-k \in \mathbf{L}^{2}(\mathbb{R})$ we deduce

$$
\phi(x)-k=\frac{1}{2 \pi} \int_{-\infty}^{\infty} \int_{-\infty}^{\infty} e^{i u x} e^{-i u z}(\phi(z)-k) d z d u .
$$

With respect to (6) we get

$$
\begin{aligned}
\pi_{t}^{Q} & =f^{Q}\left(X_{t}, t\right)=\mathbf{E}^{Q}\left[\phi\left(X_{T}\right) \mid \mathcal{F}_{t}\right] \\
& =\mathbf{E}^{Q}\left[\phi\left(X_{T}\right)-k \mid \mathcal{F}_{t}\right]+k \\
& =\frac{1}{2 \pi} \mathbf{E}^{Q}\left[\int_{-\infty}^{\infty} \int_{-\infty}^{\infty} e^{i u X_{T}} e^{-i u z}(\phi(z)-k) d z d u \mid \mathcal{F}_{t}\right]+k \\
& =\frac{1}{2 \pi} \int_{-\infty}^{\infty} \int_{-\infty}^{\infty} \mathbf{E}^{Q}\left[e^{i u X_{T}} \mid \mathcal{F}_{t}\right] e^{-i u z}(\phi(z)-k) d z d u+k \\
& =\int_{-\infty}^{\infty} \mathbf{E}^{Q}\left[e^{i u X_{T}} \mid \mathcal{F}_{t}\right] \check{\varphi}(u) d u+k,
\end{aligned}
$$

where we applied Fubini's theorem and $\check{\varphi}(\cdot)$ denotes the inverse Fourier transform of $\phi(\cdot)-k$, i.e.

$$
\check{\varphi}(u)=\frac{1}{2 \pi} \int_{-\infty}^{\infty} e^{-i u z}(\phi(z)-k) d z .
$$

Since a compound Poisson process is a Markov process with stationary and independent increments, we have

$$
\begin{aligned}
\mathbf{E}^{Q}\left[e^{i u X_{T}} \mid \mathcal{F}_{t}\right] & =e^{i u X_{t}} \mathbf{E}^{Q}\left[e^{i u\left(X_{T}-X_{t}\right)} \mid X_{t}\right] \\
& =e^{i u X_{t}} \mathbf{E}^{Q}\left[e^{i u X_{T-t}} \mid X_{t}\right] \\
& =e^{i u X_{t}} \mathbf{E}^{Q}\left[e^{i u X_{T-t}}\right] .
\end{aligned}
$$

$\mathbf{E}^{Q}\left[e^{i u X_{T-t}}\right]$ is the characteristic function of the random variable $X_{T-t}$ under the probability measure $Q$ and given by

$$
\chi_{T-t}^{Q}(u)=\exp \left(\lambda^{Q}\left(\int_{0}^{\infty} e^{i u y} d G^{Q}(y)-1\right)(T-t)\right)
$$

(see for example Karlin and Taylor [21] p.428).

Hence, the price at time $t$ of the catastrophe insurance derivative is given by

$$
\begin{aligned}
f^{Q}\left(X_{t}, t\right) & =\int_{-\infty}^{\infty} e^{i u X_{t}} \chi_{T-t}^{Q}(u) \check{\varphi}(u) d u+k \\
& =\int_{-\infty}^{\infty} e^{i u X_{t}} \exp \left(\lambda^{Q}\left(\mathbf{E}^{Q}\left[e^{i u Y_{1}}\right]-1\right)(T-t)\right) \check{\varphi}(u) d u+k
\end{aligned}
$$


The inverse Fourier transform can be explicitly calculated for the catastrophe derivatives that are traded at the CBoT, i.e. for spreads, call and put options.

This representation of no-arbitrage price processes enables us to derive the inverse Fourier transform of the price process in closed form. For a given value of the loss index $X_{t}=x$, we have

$$
\frac{1}{2 \pi} \int_{-\infty}^{\infty} e^{-i u x}\left(f^{Q}(x, t)-k\right) d x=\chi_{T-t}^{Q}(u) \cdot \check{\varphi}(u) .
$$

Our result can be summarized as follows:

Proposition 5 Let $X$ be a compound Poisson process with characteristics $\left(\lambda^{Q}, d G^{Q}(y)\right)$ under the probability measure $Q$, let $\phi: \mathbb{R} \rightarrow \mathbb{R}$ be a function such that $\phi(\cdot)-k \in \mathbf{L}^{2}(\mathbb{R})$ for some $k \in \mathbb{R}$, and let $\left(f^{Q}\left(X_{t}, t\right)\right)_{0 \leq t \leq T}$ be a stochastic process defined through

$$
f^{Q}\left(X_{t}, t\right)=\mathbf{E}^{Q}\left[\phi\left(X_{T}\right) \mid \mathcal{F}_{t}\right] .
$$

Then the function $f^{Q}: \mathbb{R}_{+} \times[0, T] \rightarrow \mathbb{R}$ defining the process $\left(f^{Q}\left(X_{t}, t\right)\right)_{0 \leq t \leq T}$ can be represented by

$$
f^{Q}(x, t)=\int_{-\infty}^{\infty} e^{i u x} \chi_{T-t}^{Q}(u) \check{\varphi}(u) d u+k,
$$

where $\check{\varphi}(\cdot)$ is the inverse Fourier transform of $\phi(\cdot)-k$ and $\chi_{X_{T-t}}^{Q}(\cdot)$ is the characteristic function of $X_{T-t}$ under the probability measure $Q$, i.e.

$$
\chi_{T-t}^{Q}(u)=\exp \left(\lambda^{Q}\left(\int_{0}^{\infty} e^{i u y} d G^{Q}(y)-1\right)(T-t)\right) .
$$

Therefore, the inverse Fourier transform of $f^{Q}(\cdot, t)-k$ is given by

$$
\frac{1}{2 \pi} \int_{-\infty}^{\infty} e^{-i u x}\left(f^{Q}(x, t)-k\right) d x=\chi_{T-t}^{Q}(u) \cdot \check{\varphi}(u) .
$$

R emark 6 It is interesting to observe that the ratio

$$
\frac{\frac{1}{2 \pi} \int_{-\infty}^{\infty} e^{-i u x}\left(\mathbf{E}^{Q}\left[\phi\left(X_{T}\right) \mid X_{t}=x\right]-k\right) d x}{\mathbf{E}^{Q}\left[e^{i u X_{T-t}}\right]}=\check{\varphi}(u)
$$

does not depend on the probability measure that we choose. Hence, for any two equivalent probability measures $Q_{1}$ and $Q_{2}$ we have

$$
\frac{\int_{-\infty}^{\infty} e^{-i u x}\left(\mathbf{E}^{Q_{1}}\left[\phi\left(X_{T}\right) \mid X_{t}=x\right]-k\right) d x}{\int_{-\infty}^{\infty} e^{-i u x}\left(\mathbf{E}^{Q_{2}}\left[\phi\left(X_{T}\right) \mid X_{t}=x\right]-k\right) d x}=\frac{\mathbf{E}^{Q_{1}}\left[e^{i u X_{T-t}}\right]}{\mathbf{E}^{Q_{2}}\left[e^{i u X_{T-t}}\right]} .
$$


One question we would like to answer is whether different equivalent probability measures will lead to different prices for a given payoff $\phi\left(X_{T}\right)$ at maturity. To be consistent with the notation used in Section 3.2, p. 9, let us characterize the equivalent probability measure $Q$ by the parameters $(\kappa, v(\cdot))$ that reflect the change in the local characteristics of the compound Poisson process $X$. Recall that the local characteristics of the process $X$ under the probability measure $Q=P^{\kappa, v} \sim P$ are given by $\lambda^{Q}=\lambda \kappa$ and $d G^{Q}(y)=v(y) d G(y)$. Let us denote the price process that corresponds to the probability measure $P^{\kappa, v}$ by $\left(f^{\kappa, v}\left(X_{t}, t\right)\right)_{0 \leq t \leq T}$, i.e. $f^{\kappa, v}$ is given by

$$
f^{\kappa, v}(x, t)=\int_{-\infty}^{\infty} e^{\left(\lambda \kappa\left(\int_{0}^{\infty} e^{i u y} v(y) d G(y)-1\right)(T-t)+i u(x-z)\right)} \check{\varphi}(u) d u+k .
$$

Lemma 7 Assume that the payoff function $\phi$ is non-constant. Then the mapping

$$
(\kappa, v(\cdot)) \in K \times V \rightarrow f^{\kappa, v} \in \mathbf{C}^{0,1}(\mathbb{R} \times[0, T])
$$

is injective where $f^{\kappa, v}$ is given by the formula (15) and

$K \times V \equiv\left\{(\kappa, v(\cdot)) \in \mathbb{R}_{+} \times \mathbf{L}^{1}\left(\mathbb{R}_{+}, G\right) \mid \mathbf{E}^{P}\left[v\left(Y_{1}\right)\right]=1\right\}$.

Proof. Assume that $f^{\kappa, v}(x, t)=f^{\kappa^{\prime}, v^{\prime}}(x, t)$ for all $x \geq 0$ and $0 \leq t \leq T$ for some $(\kappa, v(\cdot)),\left(\kappa^{\prime}, v^{\prime}(\cdot)\right) \in K \times V$. From the formula for $f^{\kappa, v}$ and $f^{\kappa^{\prime}, v^{\prime}}$ we deduce that for all $x$ and $t$

$$
\begin{aligned}
0= & \frac{1}{2 \pi} \int_{-\infty}^{\infty} \int_{-\infty}^{\infty} e^{i u(x-z)}(\phi(z)-k) \\
& \times\left(e^{\lambda \kappa \mathbf{E}^{P}\left[e^{i u Y_{1}} \cdot v\left(Y_{1}\right)-1\right](T-t)}-e^{\lambda \kappa^{\prime} \mathbf{E}^{P}\left[e^{i u Y_{1}} \cdot v^{\prime}\left(Y_{1}\right)-1\right](T-t)}\right) d z d u
\end{aligned}
$$

We observe that the double integral is the Fourier transform of

$$
\begin{gathered}
\frac{1}{2 \pi}\left(e^{\lambda \kappa \mathbf{E}^{P}\left[e^{i u Y_{1}} \cdot v\left(Y_{1}\right)-1\right](T-t)}-e^{\lambda \kappa^{\prime} \mathbf{E}^{P}\left[e^{i u Y_{1}} \cdot v^{\prime}\left(Y_{1}\right)-1\right](T-t)}\right) \\
\times \int_{-\infty}^{\infty} e^{-i u z}(\phi(z)-k) d z
\end{gathered}
$$

The Fourier transform is a one-to-one mapping of $\mathbf{L}^{2}(\mathbb{R})$ onto itself. Since it is assumed that $\phi$ is non-constant, for all $u$ and $t$ we have

$$
\lambda \kappa \mathbf{E}^{P}\left[e^{i u Y_{1}} \cdot v\left(Y_{1}\right)-1\right](T-t)=\lambda \kappa^{\prime} \mathbf{E}^{P}\left[e^{i u Y_{1}} \cdot v^{\prime}\left(Y_{1}\right)-1\right](T-t) .
$$

For $u \rightarrow \infty$ we deduce $\kappa=\kappa^{\prime}$ and hence

$$
\mathbf{E}^{P}\left[e^{i u Y_{1}} \cdot v\left(Y_{1}\right)\right]=\mathbf{E}^{P}\left[e^{i u Y_{1}} \cdot v^{\prime}\left(Y_{1}\right)\right]
$$


for all $u$. Again, since the Fourier transform is a one-to-one mapping we can conclude that

$$
v \equiv v^{\prime}
$$

This result is important as it shows that the market price of frequency risk $\kappa$ and jump size risk $v(\cdot)$ can be uniquely obtained as implied parameters from observed derivative prices. However, the result does not carry over to actuarial valuation in a similar "no-arbitrage" context as introduced by Delbaen and Haezendonck [12]. In fact there are many equivalent probability measures that lead to the same insurance premium.

Before investigating spreads, call and put options in more depth, we present an alternative method of deriving the pricing formula (11) that can be reconciled with the first method presented.

\subsubsection{Second M ethod (Pricing equation)}

This method exploits the fact that discounted price processes in the insurance market are martingales under an equivalent martingale measure. To characterize martingales based on the underlying PCS loss index $X$ we make use of the concept of an infinitesimal generator associated with a Markov process. In fact, it is possible to define the infinitesimal generator by the following martingale property (see e.g. Davis [11] for further details):

The infinitesimal generator $\mathcal{A}$ associated with a Markov process $X=\left(X_{t}\right)_{0 \leq t \leq T}$ is an operator on the set of functions $f: \mathbb{R}_{+} \times[0, T] \rightarrow \mathbb{R}$ in its domain, for which the process $M=\left(M_{t}\right)_{0 \leq t \leq T}$ with

$$
M_{t}=f\left(X_{t}, t\right)-f\left(X_{0}, 0\right)-\int_{0}^{t} \mathcal{A}(f)\left(X_{s}, s\right) d s
$$

is a martingale under $Q$. Let $\mathcal{D}(\mathcal{A})$ denote the domain of the infinitesimal generator.

The underlying PCS index $X$ is a Markov process as it is a stochastic process with stationary increments that are independent of the past. The infinitesimal generator of $X$ with local characteristics $\left(\lambda^{Q}, d G^{Q}(y)\right)$ can be represented as

$$
\mathcal{A}\left(f^{Q}\right)(x, t)=\frac{\partial}{\partial t} f^{Q}(x, t)+\lambda^{Q} \cdot \int_{0}^{\infty}\left(f^{Q}(x+y, t)-f^{Q}(x, t)\right) d G^{Q}(y),
$$

for all $f^{Q} \in \mathcal{D}(\mathcal{A})$ (see Davis [11]).

Dassios and Embrechts [10] proved that if $f$ is a measurable function, and

$$
\mathbf{E}^{Q}\left[\sum_{T_{i} \leq t}\left|f\left(X_{T_{i}}, T_{i}\right)-f\left(X_{T_{i}-}, T_{i}\right)\right|<\infty,\right.
$$


for all $0 \leq t \leq T$ then $f$ belongs to the domain of the infinitesimal generator.

Since the discounted price process of an insurance derivative is a martingale under the measure $Q$, we are interested in characterizing the set of martingales that can be constructed as a function of the underlying index $X$ for a particular contract. In the following Proposition we present a necessary and sufficient condition, in form of an integro-differential equation, for a process $\left(f^{Q}\left(X_{t}, t\right)\right)_{0 \leq t \leq T}$ to be a martingale under $Q$. This equation can also be derived by using the change of variable formula as described by Barfod and Lando [4].

Proposition 8 Let $X$ be a compound Poisson process with local characteristics $\left(\lambda^{Q}, d G^{Q}(y)\right)$ under the measure $Q$ and let $f^{Q}: \mathbb{R}_{+} \times[0, T] \rightarrow \mathbb{R}$ belong to the domain of the infinitesimal generator $\mathcal{A}$ of $X$. Then $\left(f^{Q}\left(X_{t}, t\right)\right)_{0 \leq t \leq T}$ is a martingale under $Q$ if and only if $f^{Q}$ satisfies the integro-differential equation

$$
\frac{\partial}{\partial t} f^{Q}(x, t)=\lambda^{Q} \cdot f^{Q}(x, t)-\lambda^{Q} \cdot \int_{0}^{\infty} f^{Q}(x+y, t) d G^{Q}(y),
$$

for all given values $X_{t}=x \geq 0$ and $0 \leq t \leq T$.

P roof. Suppose $f^{Q}$ satisfies the integro-differential equation (19), i.e. $\mathcal{A}\left(f^{Q}\right) \equiv 0$ by (17). Therefore, we know from (16) that $\left(f^{Q}\left(X_{t}, t\right)\right)_{0 \leq t \leq T}$ is a martingale under $Q$.

Now suppose that $\left(f^{Q}\left(X_{t}, t\right)\right)_{0 \leq t \leq T}$ is a martingale under $Q$ with mean $f^{Q}\left(X_{0}, 0\right)$. Applying the martingale property to the martingale $M$ in (16) we can deduce that the process

$$
\left(\int_{0}^{t} \mathcal{A}\left(f^{Q}\right)\left(X_{s}, s\right) d s\right)_{0 \leq t \leq T}
$$

is a zero-mean martingale under $Q$. Furthermore

$$
\begin{aligned}
& \int_{0} \mathcal{A}\left(f^{Q}\right)\left(X_{s}, s\right) d s \\
= & \int_{0}^{\cdot}\left(\frac{\partial}{\partial s} f^{Q}\left(X_{s}, s\right)+\lambda^{Q} \int_{0}^{\infty}\left(f^{Q}\left(X_{s}+y, s\right)-f^{Q}\left(X_{s}, s\right)\right) d G^{Q}(y)\right) d s
\end{aligned}
$$

is a continuous process of finite variation. Therefore, it has to be constant (see Revuz and Yor [30] p.120) and equal to zero, i.e.

$$
\int_{0}^{t}\left(\frac{\partial}{\partial s} f^{Q}\left(X_{s}, s\right)+\lambda^{Q} \int_{0}^{\infty}\left(f^{Q}\left(X_{s}+y, s\right)-f^{Q}\left(X_{s}, s\right)\right) d G^{Q}(y)\right) d s=0 .
$$

For a given value $X_{s}=x$, differentiation with respect to $t$ leads to the integro-differential equation (19).

In order to prove the uniqueness of the solution of this integro-differential equation for a given boundary condition it is useful to transform the integrodifferential equation (19) into an integral equation using variation of constants. 
Corollary $9 f^{Q}$ satisfies the integro-differential equation (19) if and only if

$$
\begin{aligned}
f^{Q}(x, t)= & e^{-\lambda^{Q}(T-t)} f^{Q}(x, T) \\
& +\lambda^{Q} \cdot \int_{t}^{T} \int_{0}^{\infty} e^{-\lambda^{Q}(s-t)} f^{Q}(x+y, s) d G^{Q}(y) d s,
\end{aligned}
$$

for $0 \leq t \leq T$ and $x \geq 0$.

Proof. Define $h^{Q}: \mathbb{R}_{+} \times[0, T] \rightarrow \mathbb{R}$ through

$$
f^{Q}(x, t)=e^{\lambda^{Q} t} \cdot h^{Q}(x, t) .
$$

Substitution into the integro-differential equation (19) leads to

$$
\frac{\partial}{\partial t} h^{Q}(x, t)=-\lambda^{Q} \cdot \int_{0}^{\infty} h^{Q}(x+y, t) d G^{Q}(y) .
$$

By integrating we obtain

$$
h^{Q}(x, t)=\lambda^{Q} \cdot \int_{t}^{T} \int_{0}^{\infty} h^{Q}(x+y, s) d G^{Q}(y) d s+h^{Q}(x, T) .
$$

Resubstitution leads to the integral equation (20) for $f^{Q}$.

In the following Proposition we provide a solution of the integro-differential equation (19) and prove uniqueness for an arbitrary but fixed boundary condition. In the context of the insurance market, we thus derive the unique price of an insurance derivative for a fixed martingale measure and payoff structure at maturity. The solution coincides with the pricing formula (11) derived through risk neutral valuation.

Proposition 10 Let $G^{Q}: \mathbb{R} \rightarrow[0,1]$ be a distribution function with support $[0, \infty), \lambda^{Q} \in \mathbb{R}_{+}$, and $\phi: \mathbb{R} \rightarrow \mathbb{R}$ be a function such that $\phi(\cdot)-k \in \mathbf{L}^{2}(\mathbb{R})$ for some $k \in \mathbb{R}$. Then the integro-differential equation

$$
\frac{\partial}{\partial t} f^{Q}(x, t)=\lambda^{Q} \cdot f^{Q}(x, t)-\lambda^{Q} \cdot \int_{0}^{\infty} f^{Q}(x+y, t) d G^{Q}(y)
$$

with the boundary condition $f^{Q}(x, T)=\phi(x)$ has the unique solution

$$
f^{Q}(x, t)=\int_{-\infty}^{\infty} \exp \left(\lambda^{Q}\left(\int_{0}^{\infty} e^{i u y} d G^{Q}(y)-1\right)(T-t)+i u x\right) \check{\varphi}(u) d u+k,
$$

in the space of all measurable functions $f^{Q}: \mathbb{R}_{+} \times[0, T] \rightarrow \mathbb{R}$ that are differentiable with respect to the second variable. $\check{\varphi}(\cdot)$ denotes the inverse Fourier transform of $\phi(\cdot)-k$, i.e.

$$
\check{\varphi}(u)=\frac{1}{2 \pi} \int_{-\infty}^{\infty} e^{-i u z}(\phi(z)-k) d z .
$$


Proof. First, we prove uniqueness by using the Gronwall inequality that states the following:

Let $v$ be a nonnegative function such that

$$
v(t) \leq C+A \cdot \int_{0}^{t} v(s) d s, \quad \text { for all } 0 \leq t \leq T,
$$

for some constants $C$ and $A$. Then

$$
v(t) \leq C \cdot \exp (A \cdot t) \quad \text { for all } 0 \leq t \leq T .
$$

Suppose now that $f_{1}^{Q}, f_{2}^{Q}: \mathbb{R}_{+} \times[0, T] \rightarrow \mathbb{C}$ are solutions of (21) with the same boundary condition $\phi$, i.e. $f_{1}^{Q}(x, T)=f_{2}^{Q}(x, T)=\phi(x)$. Define the function $h^{Q}: \mathbb{R}_{+} \times[0, T] \rightarrow \mathbb{R}$ by $h^{Q}(x, t)=\left|f_{1}^{Q}(x, t)-f_{2}^{Q}(x, t)\right|$. Then $h^{Q} \geq 0$ and by the integral representation (20) of solutions given in Corollary 9 , p. 18 we deduce that

$$
\begin{aligned}
& h^{Q}(x, t) \\
= & \left|\lambda^{Q} \cdot \int_{t}^{T} \int_{0}^{\infty} e^{-\lambda^{Q}(s-t)}\left(f_{1}^{Q}(x+y, s)-f_{2}^{Q}(x+y, s)\right) d G^{Q}(y) d s\right| \\
\leq & \lambda^{Q} \cdot \int_{t}^{T} \int_{0}^{\infty} e^{-\lambda^{Q}(s-t)} h^{Q}(x+y, s) d G^{Q}(y) d s .
\end{aligned}
$$

Let us revert time by defining the function $\bar{h}^{Q}: \mathbb{R}_{+} \times[0, T] \rightarrow \mathbb{R}$ by $\bar{h}^{Q}(x, t)=h^{Q}(x, T-t)$. Hence $\bar{h}^{Q} \geq 0$ and

$$
\begin{aligned}
\bar{h}^{Q}(x, t) & \leq \lambda^{Q} \cdot \int_{T-t}^{T} \int_{0}^{\infty} e^{-\lambda^{Q}(s-T+t)} \bar{h}^{Q}(x+y, T-s) d G^{Q}(y) d s \\
& =\lambda^{Q} \cdot \int_{0}^{t} \int_{0}^{\infty} e^{-\lambda^{Q}(t-s)} \bar{h}^{Q}(x+y, s) d G^{Q}(y) d s .
\end{aligned}
$$

Since $G^{Q}$ is a distribution function we derive for $0 \leq t \leq T$ and $x \geq 0$

$$
\begin{aligned}
\bar{h}^{Q}(x, t) & \leq \sup _{x \geq 0} \lambda^{Q} \cdot \int_{0}^{t} \bar{h}^{Q}(x, s) d s \\
& \leq \lambda^{Q} \cdot \int_{0}^{t} \sup _{x \geq 0} \bar{h}^{Q}(x, s) d s .
\end{aligned}
$$

As this inequality holds for all $x \geq 0$ it is satisfied for the supremum, i.e.

$$
\sup _{x \geq 0} \bar{h}^{Q}(x, t) \leq \lambda^{Q} \cdot \int_{0}^{t} \sup _{x \geq 0} \bar{h}^{Q}(x, s) d s, \quad \text { for all } 0 \leq t \leq T .
$$


If we define the function $v$ by $v(t)=\sup _{x \geq 0} \bar{h}^{Q}(x, t)$ we have thus shown that

$$
v(t) \leq \lambda^{Q} \cdot \int_{0}^{t} v(s) d s, \quad \text { for all } 0 \leq t \leq T
$$

and therefore condition (23) for applying the Gronwall inequality is satisfied for $C=0$ and $A=\lambda^{Q}$. From (24) we deduce that

$$
v(t)=\sup _{x \geq 0} \bar{h}^{Q}(x, t) \leq 0, \quad \text { for all } 0 \leq t \leq T .
$$

Since $\bar{h}^{Q}$ is a nonnegative function it follows that $\bar{h}^{Q} \equiv 0$ and thus $h^{Q} \equiv 0$. Given the definition of $h^{Q}$, uniqueness of the solution is proved.

Existence is proven by the explicit solution given in (22).

Given that the price can be expressed as an expected value of the real-valued random variable $\phi\left(X_{T}\right)$ (see first method) it follows that the solution (22) is a real-valued function which we may confirm as follows:

We observe that the first integral term in the solution

$$
g(x, t)=\int_{-\infty}^{\infty} \exp \left(\lambda^{Q}\left(\int_{0}^{\infty} e^{i u y} d G^{Q}(y)-1\right)(T-t)+i u x\right) \check{\varphi}(u) d u
$$

is the Fourier transform of the function

$$
\check{g}(u, t)=\exp \left(\lambda^{Q}\left(\int_{0}^{\infty} e^{i u y} d G^{Q}(y)-1\right)(T-t)\right) \cdot \check{\varphi}(u)
$$

Remember that $\check{\varphi}(u)=\frac{1}{2 \pi} \int_{-\infty}^{\infty} e^{-i u z}(\phi(z)-k) d z$ is the inverse Fourier transform of $\phi(\cdot)-k$.

In the situation in which the boundary function $\phi$ is real-valued, we know that $\check{\varphi}(-u)=\bar{\varphi}(u)$. Therefore

$$
\begin{aligned}
\check{g}(-u, t) & =\overline{\exp \left(\lambda^{Q}\left(\int_{0}^{\infty} e^{i u y} d G^{Q}(y)-1\right)(T-t)\right)} \cdot \overline{\check{\varphi}(u)} \\
& =\overline{\check{g}(u, t)} .
\end{aligned}
$$

The Fourier transform of a function with this property is real-valued. Hence we conclude that the solution (22) defines a real-valued function. 


\subsubsection{Risk Premium}

The risk premium in insurance economics is defined as the difference between the market price of an insurance contract and the expected payoff under the contract. In our analysis the financial market determines the risk premium that is thus defined as

$$
f^{Q}\left(X_{t}, t\right)-\mathbf{E}^{P}\left[\phi\left(X_{T}\right) \mid \mathcal{F}_{t}\right]=\mathbf{E}^{Q}\left[\phi\left(X_{T}\right) \mid \mathcal{F}_{t}\right]-\mathbf{E}^{P}\left[\phi\left(X_{T}\right) \mid \mathcal{F}_{t}\right]
$$

for a fixed equivalent martingale measure $Q$.

From our pricing formula (11) we conclude that the risk premium can be represented in the form

$$
\int_{-\infty}^{\infty} e^{i u X_{t}}\left(\chi_{T-t}^{Q}(u)-\chi_{T-t}^{P}(u)\right) \check{\varphi}(u) d u
$$

where $\chi_{T-t}^{Q}(u)=\exp \left(\lambda^{Q}\left(\int_{0}^{\infty} e^{i u y} d G^{Q}(y)-1\right)(T-t)\right)$ is the characteristic function of $X_{T-t}$ under the probability measure $Q$, and $\check{\varphi}(\cdot)$ is the inverse Fourier transform of $\phi(\cdot)-k$. The inverse Fourier transform of the risk premium is thus given by

$$
\left(\chi_{T-t}^{Q}(u)-\chi_{T-t}^{P}(u)\right) \cdot \check{\varphi}(u) .
$$

In the next subsections, we explicitly calculate the Fourier inverse of $\phi(\cdot)-k$ in the situation of call options, put options, and spreads. Thus under a fixed equivalent martingale measure, we will give a closed-form expression of the inverse Fourier transform of PCS option prices.

\subsubsection{Call Spreads}

A call spread on the index is a capped call option and can be created by buying a call option with strike price $K_{1}$, and selling at the same time a call option with the same maturity but with strike price $K_{2}>K_{1}$. Hence the payoff function $\phi_{C S}(x)$ depends on the index value $x$ at maturity in the following way

$$
\phi_{C S}(x)= \begin{cases}0 & \text { if } 0 \leq x \leq K_{1} \\ x-K_{1} & \text { if } K_{1}<x \leq K_{2} \\ K_{2}-K_{1} & \text { if } x>K_{2}\end{cases}
$$

As $X_{T} \geq 0$ it is sufficient that $\left(\phi_{C S}(\cdot)-k\right) \cdot \mathbf{1}_{[0, \infty)}(\cdot) \in \mathbf{L}^{2}(\mathbb{R})$ for some $k \in \mathbb{R}$ where $\mathbf{1}_{A}(\cdot)$ denotes the indicator function on a Borel set $A$. The integrability condition is satisfied for $k=K_{2}-K_{1}$ and the inverse Fourier transform is given by 


$$
\begin{aligned}
\check{\varphi}_{C S}(u) & =\frac{1}{2 \pi} \int_{0}^{\infty} e^{-i u x}\left(\phi_{C S}(x)-\left(K_{2}-K_{1}\right)\right) d x \\
& =\frac{1}{2 \pi} \frac{1}{u^{2}}\left(e^{-i u K_{2}}-e^{-i u K_{1}}+i u\left(K_{2}-K_{1}\right)\right) .
\end{aligned}
$$

Hence, under the equivalent martingale measure $Q$ the price at time $t$ of a call spread with underlying PCS index value $X_{t}=x$ and strike prices $K_{1}<K_{2}$ is

$$
\begin{aligned}
f_{C S}^{Q}(x, t)= & \int_{-\infty}^{\infty} e^{i u x} \chi_{T-t}^{Q}(u) \check{\varphi}_{C S}(u) d u+K_{2}-K_{1} \\
= & \frac{1}{2 \pi} \int_{-\infty}^{\infty} \frac{1}{u^{2}} \chi_{T-t}^{Q}(u) e^{i u x}\left(e^{-i u K_{2}}-e^{-i u K_{1}}+i u\left(K_{2}-K_{1}\right)\right) d u \\
& +K_{2}-K_{1} .
\end{aligned}
$$

Equivalently, applying the inverse Fourier transform

$$
\begin{aligned}
& \int_{-\infty}^{\infty} e^{-i u x}\left(f_{C S}^{Q}(x, t)-\left(K_{2}-K_{1}\right)\right) d x \\
= & \chi_{T-t}^{Q}(u) \cdot \frac{1}{u^{2}}\left(e^{-i u K_{2}}-e^{-i u K_{1}}+i u\left(K_{2}-K_{1}\right)\right) .
\end{aligned}
$$

Remember that $\chi_{T-t}^{Q}(u)=\exp \left(\lambda^{Q}\left(\int_{0}^{\infty} e^{i u y} d G^{Q}(y)-1\right)(T-t)\right)$ is the characteristic function of the process $X_{T-t}$ under the measure $Q$.

\subsubsection{Put Spreads}

A put spread is a capped put option and thus the payoff $\phi_{P S}$ is given by

$$
\phi_{P S}(x)= \begin{cases}K_{2}-K_{1} & \text { if } 0 \leq x \leq K_{1} \\ K_{2}-x & \text { if } K_{1}<x \leq K_{2} \\ 0 & \text { if } x>K_{2}\end{cases}
$$

We observe that $\phi_{P S}(\cdot) \cdot \mathbf{1}_{[0, \infty)}(\cdot) \in \mathbf{L}^{2}(\mathbb{R})$ and $\phi_{P S}(x)=$ $-\left(\phi_{C S}(x)-\left(K_{2}-K_{1}\right)\right)$. Therefore

$$
\begin{aligned}
\check{\varphi}_{P S}(u) & =-\check{\varphi}_{C S}(u) \\
& =-\frac{1}{2 \pi} \frac{1}{u^{2}}\left(e^{-i u K_{2}}-e^{-i u K_{1}}+i u\left(K_{2}-K_{1}\right)\right),
\end{aligned}
$$

and

$$
\begin{aligned}
f_{P S}^{Q}(x, t) & =\frac{1}{2 \pi} \int_{-\infty}^{\infty} \frac{1}{u^{2}} \chi_{T-t}^{Q}(u) e^{i u x}\left(e^{-i u K_{1}}-e^{-i u K_{2}}-i u\left(K_{2}-K_{1}\right)\right) d u \\
& =-f_{C S}^{Q}(x, t)+K_{2}-K_{1} .
\end{aligned}
$$

We have thus shown that our pricing formula fulfills the put-call parity under every equivalent martingale measure $Q$. 


\subsubsection{Call Options}

Since every PCS index is capped at either $\$ 20$ billion or $\$ 50$ billion, a call option with strike price $K$ is in fact a call spread with "lower strike price" $K_{1}=K$ and "upper strike price" $K_{2}=\$ 20$ billion or $\$ 50$ billion. Hence we can use the pricing formula for call spreads.

\subsubsection{Put Options}

A put option on a PCS index with strike price $K$ can also be understood as a put spread with "lower strike price" $K_{1}=0$ and "upper strike price" $K_{2}=K$. Due to this observation we can again apply the pricing formula for put spreads, i.e.

$$
f_{P}^{Q}(x, t)=\frac{1}{2 \pi} \int_{-\infty}^{\infty} \frac{1}{u^{2}} \chi_{T-t}^{Q}(u) e^{i u x}\left(1-e^{-i u K}-i u K\right) d u
$$

or

$$
\int_{-\infty}^{\infty} e^{-i u x} f_{P}^{Q}(x, t) d x=\chi_{T-t}^{Q}(u) \cdot \frac{1}{u^{2}}\left(1-e^{-i u K}+i u K\right)
$$

\subsubsection{Characteristic Function of Parameterized Distributions}

Let us review some parameterized distributions with support $[0, \infty)$ and their

characteristic function. We assume that the parameters are already determined under the equivalent martingale measure $Q$.

- The Gamma distribution $\Gamma(c, \gamma)$ is defined by its density function

$$
\frac{d \Gamma(c, \gamma)(y)}{d y}=\frac{c^{\gamma} e^{-c y} y^{\gamma-1}}{\Gamma(\gamma)}
$$

with mean $\gamma / c$ and variance $\gamma / c^{2}$ where $\Gamma(\cdot)$ is the Gamma function, and $0<c, \gamma<\infty$. The characteristic function is given by

$$
\int_{0}^{\infty} e^{i u y} d \Gamma(c, \gamma)(y)=\left(\frac{c}{c-i u}\right)^{\gamma} .
$$

The characteristic function of $X_{T-t}$ under $Q$ is thus,

$$
\chi_{T-t}^{Q}(u)=\exp \left(\lambda^{Q}\left(\left(\frac{c}{c-i u}\right)^{\gamma}-1\right)(T-t)\right) .
$$

- The inverse Gaussian distribution $I G(\mu, \sigma)$ has density function

$$
\frac{d I G(\mu, \sigma)(y)}{d y}=\sqrt{\frac{\sigma}{2 \pi y^{3}}} \cdot \exp \left(\frac{-\sigma(y-\mu)^{2}}{2 \mu^{2} y}\right)
$$


with mean is $\mu$ and variance $\mu^{3} / \sigma$ for $\mu \in \mathbb{R}$ and $\sigma>0$. The characteristic function is given by

$$
\int_{0}^{\infty} e^{i u y} d I G(\mu, \sigma)(y)=\exp \left(\sigma / \mu-\sqrt{(\sigma / \mu)^{2}-2 \sigma i u}\right) .
$$

Therefore,

$$
\chi_{T-t}^{Q}(u)=\exp \left(\lambda^{Q}\left(\exp \left(\sigma / \mu-\sqrt{(\sigma / \mu)^{2}-2 \sigma i u}\right)-1\right)(T-t)\right) .
$$

- The distribution Pareto mixtures of exponentials $P M E(\delta)$ belongs to the class of distributions with heavy tails. Their density function is given by

$$
\frac{d P M E(\delta)}{d y}=\int_{(\delta-1) / \delta}^{\infty} \delta^{-\delta+1}(\delta-1)^{\delta} z^{-(\delta+1)} z^{-1} e^{-y / z} d z
$$

with mean 1 and variance $1+2 / \delta(\delta-2)$ for $\delta>1$. The characteristic function is given by

$$
\int_{0}^{\infty} e^{i u y} \operatorname{PME}(\delta)(y)=\frac{\int_{0}^{\frac{\delta}{\delta-1}} \frac{z^{\delta}}{z-i u} d z}{\frac{\delta^{\delta-1}}{(\delta-1)^{\delta}}}
$$

Therefore,

$$
\chi_{T-t}^{Q}(u)=\exp \left(\lambda^{Q}\left(\frac{\int_{0}^{\frac{\delta}{\delta-1}} \frac{z^{\delta}}{z-i u} d z}{\frac{\delta^{\delta-1}}{(\delta-1)^{\delta}}}-1\right)(T-t)\right) .
$$

In this paragraph, we investigated the valuation of catastrophe insurance derivatives for an arbitrary but fixed equivalent martingale measure. However, in the setup of our insurance market there exist an infinite number of equivalent martingale measures, and hence an infinite collection of prices that are consistent with the no-arbitrage assumption on the bond market. Therefore, we need to be more specific on the preferences of market participants. We follow an approach suggested by Aase [1] and [2] who uses the framework of partial equilibrium theory under uncertainty. The next section includes a brief outline of the economic theory as it is presented in Duffie [14], Chapter 10. 


\subsection{Representative A gent's Valuation}

Let us characterize the insurance companies $i=1,2, \ldots, I$ that are affected by catastrophes under a specific PCS contract by net reserves $S^{i}=\left(S_{t}^{i}\right)_{t \geq 0}$ and utility functions $U^{i}: L_{+} \rightarrow \mathbb{R}$ defined on the consumption space $L_{+}$. We assume that $L_{+}$is the set of nonnegative, adapted processes $C$ with $\mathbf{E}^{P}\left[\int_{0}^{T} C_{t}^{2} d t\right]<\infty$ and smooth-additivity of utility functions, i.e.

$$
U^{i}\left(C^{i}\right)=\mathbf{E}^{P}\left[\int_{0}^{T} u^{i}\left(C_{t}^{i}, t\right) d t\right],
$$

for $C^{i} \in L_{+}$. Furthermore, smooth-additivity requires that for all $i \in\{1,2, \ldots, I\}$ $u^{i}: \mathbb{R}_{+} \times[0, T] \rightarrow \mathbb{R}$ is smooth on $(0, \infty) \times[0, T]$ and, for each $0 \leq t \leq T$, $u^{i}(\cdot, t): \mathbb{R}_{+} \rightarrow \mathbb{R}$ is increasing, strictly concave, with an unbounded derivative $u_{c}^{i}(\cdot, t)=\frac{\partial}{\partial c} u^{i}(\cdot, t)$ on $(0, \infty)$.

An Arrow-Debreu equilibrium is a collection $\left(\Pi, C^{1}, C^{2}, \ldots, C^{I}\right)$ such that $C^{i}$ solves insurance company $i$ 's maximization problem

$$
\sup _{C \in L_{+}} U^{i}(C) \quad \text { subject to } \Pi(C) \leq \Pi\left(S^{i}\right),
$$

where $\left(C^{1}, C^{2}, \ldots, C^{I}\right)$ is a feasible allocation, i.e. $\sum_{i=1}^{I} C^{i} \leq \sum_{i=1}^{I} S^{i} \equiv S$, where $S$ is the aggregated net reserves, and $\Pi: L \rightarrow \mathbb{R}$ is a linear price function that describes the price at time 0 for a consumption process in $L$. Furthermore, if $\Pi$ is strictly increasing, then there is a unique, strictly positive process $\pi \in L_{+}$ such that

$$
\Pi(C)=\mathbf{E}^{P}\left[\int_{0}^{T} \pi_{t} C_{t} d t\right] \quad \text { for } C \in L .
$$

Since $U^{i}$ is strictly increasing any Arrow-Debreu equilibrium price function $\Pi$ is strictly increasing. The representation (35) is known as the Riesz representation of $\Pi(\cdot)$ (see Duffie [14] p. 221).

For incomplete markets, there is yet no set of conditions that is sufficient for the existence of an Arrow-Debreu equilibrium. However, with negative exponential utility functions a Pareto efficient outcome can be achieved and is characterized by a linear risk-sharing rule. This implies that every investor holds a certain fraction of the aggregate risk.

Let us therefore assume that preferences of investors can be described by negative exponential utility functions, i.e.

$$
u_{c}^{i}(c, t)=e^{-\alpha^{i} c-\rho^{i} t},
$$

for some $\alpha^{i}>0, \rho^{i}>0 . \alpha^{i}$ represents the intertemporal coefficient of absolute risk aversion and $\rho^{i}$ the time impatience rate of agent $i$. 
Under these assumptions, there exists a representative agent in the market with utility function

$$
U(C)=\mathbf{E}^{P}\left[\int_{0}^{T} u\left(C_{t}, t\right) d t\right]
$$

where $u$ is of the form

$$
u_{c}(c, t)=e^{-\alpha c-\rho t},
$$

with intertemporal coefficient of absolute risk aversion $\alpha>0$ and time impatience rate $\rho>0$ in the market. Furthermore, the Riesz representation $\pi$ of $\Pi(\cdot)$ is given by

$$
\pi_{t}=u_{c}\left(S_{t}, t\right) \text {, }
$$

with aggregated net reserves $S_{t}=\sum_{i=1}^{I} S^{i}$.

Coming back to the martingale approach, $\pi$ is not only the Riesz representation of $\Pi$ but also the gradient of $U$ (see Duffie [14] p. 300) and defines a state-price deflator. Furthermore, this state-price deflator determines an equivalent martingale measure $Q$ through the Radon-Nikodym density process

$$
\begin{aligned}
\xi_{t} & =\exp \left(\int_{0}^{t} r_{u} d u\right) \cdot \frac{\pi_{t}}{\pi_{0}} \\
& =\exp \left(\int_{0}^{t} r_{u} d u\right) \cdot \frac{u_{c}\left(S_{t}, t\right)}{u_{c}\left(S_{0}, 0\right)} .
\end{aligned}
$$

In addition, we know from the last section (see (3)) that $\xi$ can be represented by

$$
\xi_{t}=\exp \left(\sum_{k=1}^{N_{t}} \ln \left(\kappa v\left(Y_{k}\right)\right)+\int_{0}^{t} \int_{0}^{\infty}(1-\kappa v(y)) \lambda d G(y) d s\right),
$$

for some nonnegative constant $\kappa$ and nonnegative function $v$.

R emark 11 This equivalent martingale measure $Q=P^{\kappa, v}$ can be interpreted as the one under which the representative agent calculates prices in the insurance market. Hence, the corresponding local characteristics $\kappa$ and $v(\cdot)$ reflect the representative agent's market price of frequency risk and claim size risk respectively.

We follow the classical Cramér-Lundberg model and assume that aggregate net reserves in the insurance industry is represented by a stochastic process $S=\left(S_{t}\right)_{0 \leq t \leq T}$ of the form 


$$
\begin{aligned}
S_{t} & =s_{0}+p t-X_{t} \\
& =s_{0}+p t-\sum_{k=1}^{N_{t}} Y_{k},
\end{aligned}
$$

where $s_{0}$ represents aggregate initial capital in the market by time $0, X_{t}$ is the PCS index at time $t$ for a specific contract, and $p$ is total premium of the industry for a unit time interval within the loss period of the contract. Hence, the process $S$ represents the surplus of those companies that are affected by catastrophe losses reflected in the particular PCS index $X$. For example, the net reserves of an insurance company in Florida would not be included if we consider the California index.

By equating the two representations (40) and (41) of $\xi$ and putting $r \equiv 0$, we deduce

$$
-(\alpha p+\rho) t+\sum_{k=1}^{N_{t}} \alpha Y_{k}=\lambda(1-\kappa) t+\sum_{k=1}^{N_{t}} \ln \left(\kappa v\left(Y_{k}\right)\right),
$$

for $0 \leq t \leq T$. Therefore

$$
\kappa v(y)=e^{\alpha y},
$$

for $y \geq 0$. Since $\int_{0}^{\infty} v(y) d G(y)=1$

$$
\begin{aligned}
\kappa & =\mathbf{E}^{P}\left[e^{\alpha Y_{1}}\right] \\
v(y) & =\frac{e^{\alpha y}}{\mathbf{E}^{P}\left[e^{\alpha Y_{1}}\right]} .
\end{aligned}
$$

Additionally, equation (43) imposes the following restriction on the parameters of the model:

$$
\alpha p+\rho=\lambda\left(\mathbf{E}^{P}\left[e^{\alpha Y_{1}}\right]-1\right) .
$$

This leads to the following corollary:

Corollary 12 Consider a market containing a risk averse representative agent as outlined above. Then the coefficient of absolute risk aversion $\alpha$ is uniquely determined by the equilibrium relation (44) for a given premium rate $p$ and time impatience rate $\rho$ in the market.

Proof. We only consider risk aversion, i.e. we assume $\alpha>0$. The same argument holds for a risk loving agent. We have to prove the existence of a unique $\alpha^{*}>0$ satisfying (44).

Define the function $h: \mathbb{R}_{+} \rightarrow \mathbb{R}$ by

$$
h(\alpha)=\lambda\left(\mathbf{E}^{P}\left[e^{\alpha Y_{1}}\right]-1\right)-\alpha p-\rho,
$$

for given $p, \rho>0$. We deduce $h(0)=-\rho<0$ and 


$$
\frac{d^{2}}{d \alpha^{2}} h(\alpha)=\lambda \mathbf{E}^{P}\left[Y_{1}^{2} e^{\alpha Y_{1}}\right]>0,
$$

i.e. $h$ is a convex function.

If the distribution function $G$ is sufficiently regular then

$$
h(\alpha) \rightarrow+\infty \quad \text { for } \alpha \rightarrow \infty,
$$

and there exists a unique $\alpha^{*}>0$ such that $h\left(\alpha^{*}\right)=0$.

Alternatively, for a given degree of absolute risk aversion $\alpha$ the premium rate $p$ is of the form

$$
p=\frac{1}{\alpha}\left(\lambda\left(\mathbf{E}^{P}\left[e^{\alpha Y_{1}}\right]-1\right)-\rho\right) .
$$

The first factor $1 / \alpha$ reflects the representative agent's risk tolerance whereas the second can be interpreted as the difference between the frequency risk premium $\lambda\left(\mathbf{E}^{P}\left[e^{\alpha Y_{1}}\right]-1\right)$ and the time impatience rate $\rho$. The agent's risk tolerance and frequency risk premium are positively related to the premium rate $p$ contrary to the time impatience rate.

R emark 13 Under risk aversion, i.e. $\alpha>0$ we observe that $\kappa v(y)>1$ for all $y>0$. As $v($.$) is a density, it follows that \kappa>1$. We conclude that in a risk-averse insurance market the risk-adjusted frequency $\lambda \kappa$ is larger than the physical frequency $\lambda$.

The coefficient of absolute risk aversion $\alpha$ determines uniquely the market prices of frequency risk $\kappa$ and of jump size risk $v(\cdot)$ and thus the equivalent martingale measure $P^{\kappa, v}=P^{\alpha}$, the local characteristics of the underlying PCS loss index under $P^{\alpha}$, and the price process of catastrophe insurance derivatives as follows:

- prices are calculated under the equivalent measure $P^{\alpha} \sim P$ that is defined through its density process

$$
\begin{aligned}
\xi_{t} & =\exp \left(\sum_{k=1}^{N_{t}} \alpha Y_{k}+\int_{0}^{t} \int_{0}^{\infty}\left(1-e^{\alpha y}\right) \lambda d G(y) d s\right) \\
& =\exp \left(\alpha X_{t}+\int_{0}^{t} \int_{0}^{\infty}\left(1-e^{\alpha y}\right) \lambda d G(y) d s\right)
\end{aligned}
$$

- $X$ is a compound Poisson process under $P^{\alpha}$ with local characteristics

$$
\begin{aligned}
\mathbf{E}^{P^{\alpha}}\left[N_{1}\right] & =\lambda \cdot \mathbf{E}^{P}\left[e^{\alpha Y_{1}}\right] \\
d G^{\alpha}(y) & =\frac{e^{\alpha y}}{\mathbf{E}^{P}\left[e^{\alpha Y_{1}}\right]} d G(y),
\end{aligned}
$$


- the unique price process $\left(f^{\alpha}\left(X_{t}, t\right)\right)_{0 \leq t \leq T}$ of an insurance derivative with payoff function $\phi$ is given by

$$
f^{\alpha}\left(X_{t}, t\right)=\int_{-\infty}^{\infty} e^{i u X_{t}} \chi_{X_{T-t}}^{P^{\alpha}}(u) \check{\varphi}(u) d u+k
$$

where

$$
\chi_{T-t}^{P^{\alpha}}(u)=\exp \left(\lambda \int_{0}^{\infty} e^{\alpha y}\left(e^{i u y}-1\right) d G(y)(T-t)\right),
$$

and $\check{\varphi}(\cdot)$ is the inverse Fourier transform of $\phi(\cdot)-k$.

The parameters of the model are restricted by the equilibrium relation

$$
\alpha p+\rho=\lambda\left(\mathbf{E}^{P}\left[e^{\alpha Y_{1}}\right]-1\right) .
$$

Let us finish this section with the following remark.

R emark 14 For given parameters $\alpha, p$, and $\rho$ the characteristic function is of the form

$$
\chi_{T-t}^{P^{\alpha}}(u)=\exp \left(\frac{\mathbf{E}^{P}\left[e^{\alpha Y_{1}}\left(e^{i u Y_{1}}-1\right)\right]}{\mathbf{E}^{P}\left[e^{\alpha Y_{1}}-1\right]} \cdot(\alpha p+\rho) \cdot(T-t)\right) .
$$

We have thus established a link between the premium rate $p$ and the price process $\left(f^{\alpha}\left(X_{t}, t\right)\right)_{0 \leq t \leq T}$ through the characteristic function.

\section{Conclusion}

In this paper we examined the valuation of catastrophe insurance derivatives in a model in which the underlying, non-traded loss index is a compound Poisson process, a stochastic process used to describe aggregate losses in risk theory. Initially, we only imposed the absence of arbitrage strategies and showed how to structure the market's incompleteness by exploiting the fact that prices under specific probability measures are martingales. This structure was built on parameters that capture the market prices of frequency and loss size risk.

We introduced a new technique based on Fourier analysis that allowed us to deduce a representation of the set of no-arbitrage price processes. This representation enabled us to derive the inverse Fourier transform of derivative prices in closed form and to separate the underlying stochastic environment from the derivative's payoff structure. Furthermore, it was shown that the set of noarbitrage prices and the set of market prices of frequency and loss size risk is one-to-one connected. 
In the preference based equilibrium model the utility function of a representative agent determines uniquely the market prices of frequency and jump size risk. Building upon our representation of derivative prices and their link to market prices of risk, we determined the agent's attitude towards catastrophic risk and thus the unique price for the representative agent.

The analysis and results developed in this paper suggest to calibrate the model to market data, i.e. to obtain the market prices of risk as implied parameters from observed derivative prices. Since we derived the inverse Fourier transform of derivative prices in closed form, it is moreover suggested that there is much to be gained by using Fast Fourier Transform as an efficient algorithm for the calculation of prices.

A cknowledgements. The author would like to thank Eric Ben-Hamou, Tomas Bjørk, Markus Brunnermeier, Lucien Foldes, Rüdiger Frey, Søren Johansen, Pierre Mella-Barral, Michael Mürmann, Sönje Reiche, Dieter Sondermann, and Alexander Stremme for fruitful comments.

\section{R eferences}

[1] Aase, K. (1995). "Catastrophe Insurance Futures Contracts", Norwegian School of Economics and Business Administration, Institute of Finance and Management Science, Working Paper 1/95.

[2] Aase, K. (1999). "An Equilibrium Model of Catastrophe Insurance Futures and Spreads", Geneva Papers on Risk and Insurance Theory 24, 69-96.

[3] Balbás, A., I. R. Longarela, J. J. Lucia (1999). "How Financial Theory Applies to Catastrophe-Linked Derivatives - An Empirical Test of Several Pricing Models", The Journal of Risk and Insurance 66:4, 551-582.

[4] Barfod, A. M., and D. Lando (1996). "On Derivative Contracts on Catastrophe Losses", University of Copenhagen, Institute of Mathematical Statistics, Working Paper. 
[10] Dassios, A., and P. Embrechts (1989). "Martingales and Insurance Risk", Communications in Statistical and Stochastic Models 5, 181-217.

[11] Davis, M. H. A. (1993). Markov Models and Optimization. Monographs on Statistics and Applied Probability 49. Chapman \& Hall, London.

[12] Delbaen, F., and J. Haezendonck (1989). "A Martingale Approach to Premium Calculation Principles in an Arbitrage-free Market", Insurance: Mathematics and Economics 8, 269-277.

[13] Delbaen, F., and W. Schachermayer (1997). "Non-Arbitrage and the Fundamental Theorem of Asset Pricing: Summary of Main Results", Proceedings of Symposia in Applied Mathematics, Volume 00.

[14] Duffie, D. (1996). Dynamic Asset Pricing Theory. (2nd ed.) Princeton University Press, Princeton.

[15] Embrechts, P., and S. Meister (1997). "Pricing Insurance Derivatives, the Case of CAT-Futures", Proceedings of the 1995 Bowles Symposium on Securitization of Risk, George State University Atlanta, Society of Actuaries, Monograph M-FI97-1, 15-26.

[16] Fritelli, M., and P. Lakner (1995). "Arbitrage and Free Lunch in a General Financial Market Model; The Fundamental Theorem of Asset Pricing", in M. H. A. Davis et al. (eds), Mathematical Finance. The IMA Volumes in Mathematics and its Applications Volume 65. Springer-Verlag, Berlin, Heidelberg, New York.

[17] Gasquet, C., and P. Witomski (1999). Fourier Analysis and Applications. (translated by R. Ryan) Texts in Applied Mathematics 30. Springer-Verlag, Berlin, Heidelberg, New York.

[18] Geman, H., and M. Yor (1997). "Stochastic Time Changes in Catastrophe Option Pricing", Insurance: Mathematics and Economics 21, 185-193.

[19] Harrison, J. M., and D. M. Kreps (1979). "Martingales and Arbitrage in Multiperiod Securities Markets", Journal of Economic Theory 20, 381-408.

[20] Harrison, J. M., and S. R. Pliska (1981). "Martingales and Stochastic Integrals in the Theory of Continuous Trading", Stochastic Processes and their Applications 11, 215-260.

[21] Karlin, S., and H. M. Taylor (1981). A Second Course in Stochastic Processes. Academic Press, New York.

[22] Klüppelberg, C., and T. Mikosch (1997). "Large Deviations of Heavy-tailed Random Sums with Applications in Insurance and Finance", Journal of Applied Probability 34, 293-308.

[23] Levi, Ch., and Ch. Partrat (1991). "Statistical Analysis of Natural Events in the United States", ASTIN Bulletin 21:2, 253-276. 
[24] Mann, S. V., and G. Niehaus (1992). "The Trading of Underwriting Risk; an Analysis of Insurance Futures Contracts and Reinsurance", The Journal of Risk and Insurance 59, 601-627.

[25] Meister, S. (1995). "Contributions to the Mathematics of Catastrophe Insurance Futures", ETH Zürich, Department of Mathematics, Diplomarbeit.

[26] Merton, R. (1973). "Theory of Rational Option Pricing", Bell Journal of Economics and Management Science 4, 141-183.

[27] Musiela, M., and M. Rutkowski (1997). Martingale Methods in Financial Modelling. Applications of Mathematics 36. Springer-Verlag, Berlin, Heidelberg, New York.

[28] O’Brien, T. (1997). "Hedging Strategies Using Catastrophe Insurance Options", Insurance: Mathematics and Economics 21, 153-162.

[29] Protter, P. (1995). Stochastic Integration and Differential Equations. (3rd ed.) Applications of Mathematics 21. Springer-Verlag, Berlin, Heidelberg, New York.

[30] Revuz, D., and M. Yor (1999). Continuous Martingales and Brownian Motion. (3rd ed.) Grundlehren Math. Wiss. Bd. 293. Springer-Verlag, Berlin, Heidelberg, New York.

[31] Sondermann, D. (1991). "Reinsurance in Arbitrage-free Markets", Insurance: Mathematics and Economics 10, 191-202. 